\title{
Dewetting of thin-film polymers
}

\author{
F. Saulnier, 用 E. Raphaël, 用 and P.-G. de Gennesf \\ Laboratoire de Physique de la Matière Condensée, CNRS UMR 7125, Collège de France \\ 11, place Marcelin Berthelot, 75231 Paris Cedex 05, France.
}

(Dated: 21th of june, 2002)

\begin{abstract}
In this paper we present a theoretical model for the dewetting of ultra-thin polymer films. Assuming that the shear-thinning properties of these films can be described by a Cross-type constitutive equation, we analyze the front morphology of the dewetting film, and characterize the time evolution of the dry region radius, and of the rim height. Different regimes of growth are expected, depending on the initial film thickness, and on the power-law index involved in the constitutive equation. In the thin-films regime, the dry radius and the rim height obey power-law time dependences. We then compare our predictions with the experimental results obtained by Debrégeas et al. [Phys. Rev. Lett. 75, 3886 (1995)] and by Reiter [Phys. Rev. Lett. 87, 186101 (2001)].

PACS numbers: 68.60.-p, 68.15.+e, 68.55.-a, 83.10.-y
\end{abstract}

\section{INTRODUCTION}

Thin liquid films have considerable scientifical and technological importance, and have numerous applications. In engineering, for instance, they serve to protect surfaces, and applications arise in paints, adhesives and membranes [1]. Thin liquids films display a variety of interesting dynamics phenomena and have therefore been the focus of many experimental and theoretical studies [2]. In particular, thin polymer films have recently attracted a lot of interest since understanding their properties such as viscosity [3], chain mobility [4] and stability [5] is essential for optimization.

A long time ago, Taylor [6] and Culick [7] analyzed the growth of a circular hole in a thin liquid sheet 8]. By balancing surface tension forces against inertia [9], they found that the rim of liquid at the edge of the films retracts at a constant velocity, a prediction first checked experimentally by Mc Entee and Mysels 110, 11. Debrégeas and collaborators 12 have recently studied the bursting of thin suspended films of very viscous liquids. These experiments revealed unexpected features: (a) First, the retraction velocity grows exponentially with time (with a characteristic time scale $\tau_{i}=h_{i} \eta /|S|$, where $h_{i}, \eta$ and $S$ are respectively the initial film thickness, the viscosity and the spreading coefficient [13]), (b) second, the liquid is not collected into a rim and the film remains flat through the retraction. According to these authors, the uniform thickening of the retracting film was a consequence of its viscoelasticity, which permits an elastic propagation into the film of the surface tension forces acting on the edge. Brenner and Gueyffier [14 showed, however, that the absence of rim can also result from a purely viscous effect [15, 16].

Very recently [17, Reiter studied the dewetting of ul-

\footnotetext{
*Electronic address: florent.saulnier@college-de-france.fr

$\dagger$ Electronic address: elie.raphael@college-de-france.fr

$\ddagger$ Electronic address: pgg@espci.fr
}

trathin (i.e. thinner than the coil size), almost glassy polystyrene (PS) films deposited onto silicon wafers coated with a polydimethylsiloxane (PDMS) monolayer. He found that a highly asymmetric rim, with an extremely steep side towards the interior of the hole and a much slower decay on the rear side, builds up progressively [18], with the maximum height increasing linearly with the diameter of the hole. We recently 19 proposed a theoretical model to explain such deviations from the behavior of pure liquids, based on the shearthinning properties of the polymer film. Assuming that the stresses inside the film saturate logarithmically with the strain rates, we showed that different regimes of growth are expected, depending on the initial film thickness and the experimental time range. Other theoretical approaches for polymer film dewetting have recently been proposed by Herminghaus et al. 20] and Shenoy et al [21].

In this paper we aim at precising our methods of resolution, and characterizing the dewetting process for a Cross-type constitutive equation (interpolation between a viscous behavior at low strain-rates and a power-law dependence for the viscosity versus the shear strain rate), following recent experimental results by Dalnoki-Veress et al. for PS films 22. We study the profile of the dewetting film, and characterize the time evolution of the dry radius, and of the rim height (as summarized in appendix Q). Finally, we discuss the adequation between our theoretical predictions and experimental results by Debrégeas and Reiter.

\section{THE MODEL}

Figure 11 shows the film geometry. $h(r, t)$ is the profile of the film, $h_{m}(t)$ is the height of the rim, and $R_{d}(t)$ is the radius of the dry zone. $v(r, t)$ is the radial, axisymmetric flow field (cf. appendix A). On a non-wettable, smooth and passive solid substrate like the PDMS-coated silicon wafer used by Reiter, this plug-flow description is valid as long as $h_{i} \ll b$ where $b$ is the hydrodynamic extrapolation 


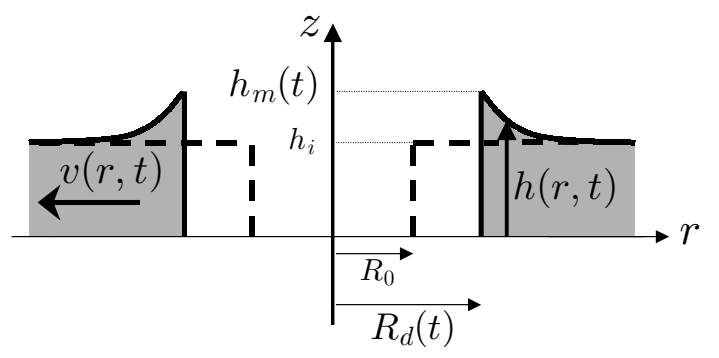

FIG. 1: Film geometry: $h(r, t)$ is the profile of the film, $h_{m}(t)$ is the height of the rim, and $R_{d}(t)$ is the radius of the dry zone. The initial $(t=0)$ step-like profile is represented by the dashed line. $v(r, t)$ is the radial, axisymmetric flow field.

length (cf. 23]). The range of thicknesses studied by Reiter allows this simplifying assumption.

\section{A. Constitutive law of the material}

In order to characterize the rheologic properties of the film, we introduce the stress tensor $\sigma_{i j}=-p \delta_{i j}+\sigma_{i j}^{m}$, where $p$ is the pressure and $\sigma_{i j}^{m}$ represents the effects of internal friction. We relate $\sigma_{i j}^{m}$ to the strain rate tensor $\dot{\gamma}_{i j}$ by a constitutive law of the form:

$$
\sigma^{m}(\dot{\gamma})=\sigma_{0} \Phi(\dot{\gamma} \tau)
$$

where $\sigma_{0}$ and $\tau$ are material constants, and $\Phi$ is a generic function.

For a purely viscous liquid, the function $\Phi$ is linear:

$$
\Phi(\dot{\gamma} \tau)=\dot{\gamma} \tau
$$

In this case, the fluid viscosity $\eta=\sigma^{m} / \dot{\gamma}$ is constant and equals the zero-shear viscosity $\eta_{0}=\sigma_{0} \tau$.

For polymers, just above $T_{g}$, it is expected within the framework of the free-volume model that $\sigma^{m}$ is expected to vary logarithmically with $\dot{\gamma}$ as 24]:

$$
\Phi(\dot{\gamma} \tau)=\ln (1+\dot{\gamma} \tau)
$$

The features of the dewetting regimes obtained with this rheological law were presented elsewhere [19], and will only be briefly mentioned in the present paper in Appendix B. As mentioned in [19], this logarithmic law does not permit a simple analytical description of all the regimes of time and thicknesses.

An alternative way of describing the polymer rheology is to use the well-known Cross model [25]:

$$
\Phi(\dot{\gamma} \tau)=\frac{\dot{\gamma} \tau}{1+k(\dot{\gamma} \tau)^{n}},
$$

$k$ and $n$ being dimensionless constant parameters. A shear-thinning behavior is taken into account by taking the power-law index $n$ strictly between 0 and 1 . At low strain-rates $\left(\dot{\gamma} \tau<k^{-1 / n}\right)$, this law displays a viscoustype behavior $\left(\sigma^{m} \approx \eta_{0} \dot{\gamma}\right.$, with a zero-shear viscosity $\left.\eta_{0}=\sigma_{0} \tau\right)$, while for large values of $\dot{\gamma} \tau, \sigma^{m}$ obeys the well-known power-law model. This popular expression, which will be used all along with this article, is applicable to a number of polymer materials and complex fluids [26].

\section{B. Equations}

Assuming the fluid to be incompressible, mass conservation leads to:

$$
\frac{1}{h(r, t)}\left(\frac{\partial h(r, t)}{\partial t}+\frac{r \beta(r, t)}{\tau} \frac{\partial h(r, t)}{\partial r}\right)=\frac{\alpha(r, t)-\beta(r, t)}{\tau}
$$

Equation 5 involves two unknown functions $\alpha(r, t)$ and $\beta(r, t)$, that are positive, dimensionless forms of the strain-rate components $\dot{\gamma}_{r r}$ and $\dot{\gamma}_{\phi \phi}$ :

$$
\left\{\begin{array}{l}
\alpha=-\tau \dot{\gamma}_{r r}=-\tau \frac{\partial v}{\partial r} \\
\beta=\tau \dot{\gamma}_{\phi \phi}=\tau \frac{v}{r}
\end{array}\right.
$$

We thus need two additional equations to determine $h(r, t)$. First, note that the following partial differential equation can be directly derived from Eq.6:

$$
\frac{\partial \beta}{\partial r}=-\frac{\beta+\alpha}{r}
$$

Neglecting the inertial term, conservation of momentum (projected on the radial direction [27) leads to:

$$
\frac{\partial \sigma_{r r}}{\partial r}+\frac{\sigma_{r r}-\sigma_{\phi \phi}}{r}=0
$$

It can be shown that Eqs. 7 and 8 , along with the freesurface boundary condition (i.e. $\sigma_{z z}=0$ at the contact with ambient atmosphere [28]), allow one to express the strain rate $\alpha$ as a function of $\beta$ only : $\alpha=F(\beta)$. Substitution of the shear-thinning constitutive law 1 in Eq.8 then leads to the following differential equation for $F$ :

$$
\frac{\mathrm{d}[\Phi(F(\beta))-\Phi(F(\beta)-\beta)]}{\mathrm{d} \beta}=\frac{\Phi(F(\beta))-\Phi(\beta)}{F(\beta)+\beta},
$$

which has to be solved along with the condition $F(0)=$ 0 (far away from the perturbed central region, the strain rates must decrease to zero). In Fig.2, we present the form of functions $\alpha=F(\beta)$ for the Cross model, taking for each curve $k=10$ and different values of power-law index $n$ between 0 and 1. This function can be approached by simpler expressions in the two opposite regimes $\beta \ll 1$ and $\beta \gg 1$, as explained below (cf.III A 1). 


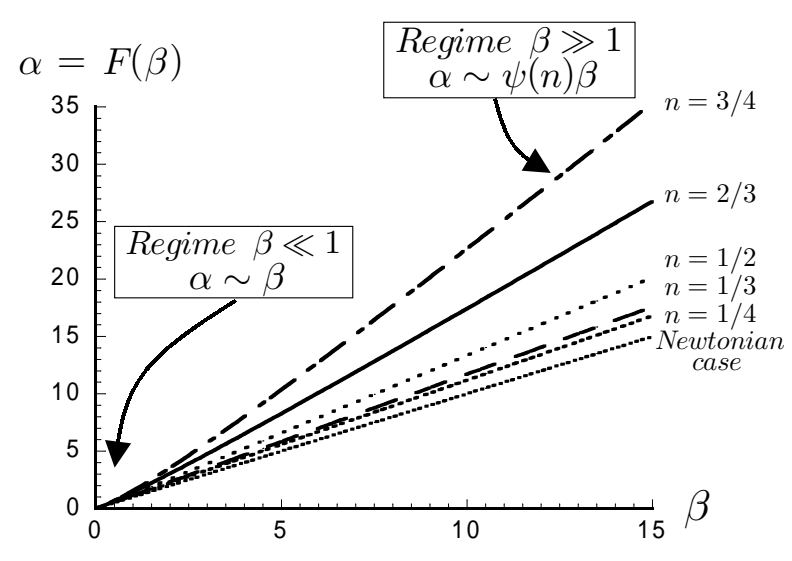

FIG. 2: The radial strain-rate, $\alpha$, and the orthoradial strainrate, $\beta$, are related by $\alpha=F(\beta)$ (see Eq.9). In the purely Newtonian case $(\Phi(\dot{\gamma} \tau)=\dot{\gamma} \tau)$, the function $F$ is linear: $\alpha=\beta$. The other curves represent $F$ for the Cross model (Eq.4), taking different values of $n$ for a given value of $k=10$ : $n=1 / 4 ; 1 / 3 ; 1 / 2 ; 2 / 3$ (an important case, as precised in part IVB); and $n=3 / 4$. The two limit regimes $\beta \ll 1$ and $\beta \gg 1$, and the corresponding approached expressions for $F$ are also represented.

In order to solve Eq. F, we should supply it with initial and boundary conditions. Our initial profile is assumed to be uniform, with a thickness $h_{i}$, except in a bored region ranging from $r=0$ to $r=R_{0}$ (as shown in fig.1). $R_{0}$ is our characteristic radial length used thereafter to make $r$ dimensionless:

$$
h(r, t=0)= \begin{cases}h_{i} & \text { if } r \geq R_{0}=1 \\ 0 & \text { otherwise }\end{cases}
$$

We do not consider here the origin of the initial dewetting process: experimentally, it is found that a thick PS film on a silicon substrate is metastable and dewets via nucleation and growth of dry patches [29], while thinner films $\left(h_{i}<100 \mathrm{~nm}\right)$ are unstable and dewet by spinodal decomposition [30, 31]. In his latest experiments on ultra-thin films [17], Reiter characterized the early stage of the dewetting process by the formation and coalescence of little holes, with the displaced material uniformly distributed between the holes, without visible rims. Our initial time $t=0$ might correspond to the end of this preliminary process.

Equation 5 applies outside the dewetted region $\left(R_{d}(t) \leq r<\infty\right)$. At the edge of the $\operatorname{rim}\left(r=R_{d}(t)\right)$, the rim height, $h_{m}(t)$, can be determined by taking into account capillary forces. The applied force on the rim, pushing the film away the dry area, must be balanced by the internal radial stress: $|S|=\left|\sigma_{r r}\right| h_{m}(t)$. Assuming the lateral extension of the film to be large enough, the film thickness must reach $h_{i}$ far from the dry region: $\lim _{r \rightarrow \infty} h(r, t)=h_{i}(\forall t)$. The complete resolution of our set of equations 5 자. 9 can be achieved using a method of characteristics (cf. appendix D).

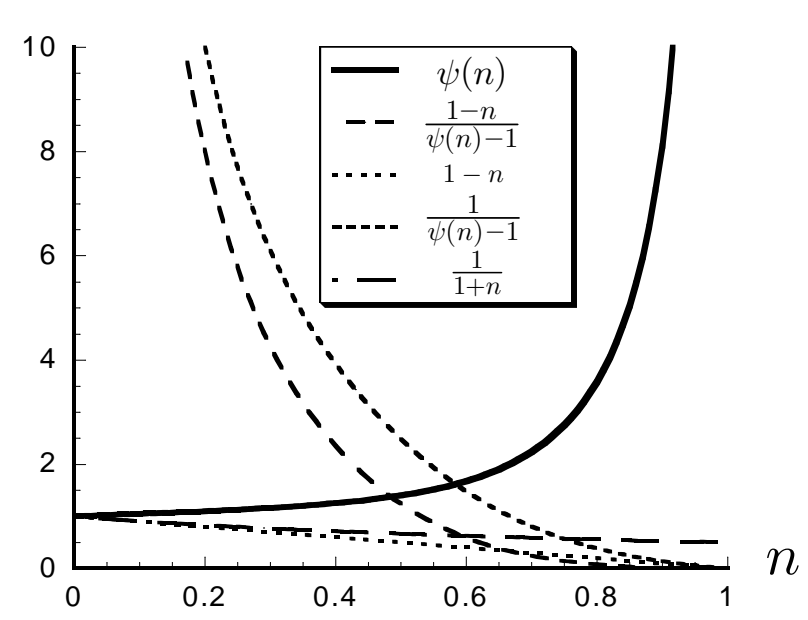

FIG. 3: Variation with the power-law index $n$ of the parameter $\psi(n)$. Some other quantities of interest are also represented (see text).

Thereafter, all thicknesses will be made dimensionless by normalizing with a characteristic length $h^{*} \equiv|S| / \sigma_{0}$. For Reiter's experimental conditions, at $T \approx 105^{\circ} \mathrm{C}$, we estimate $h^{*}$ to be of the order of $500 \AA$. This parameter $h^{*}$ is of crucial importance in our model, because it discriminates two regimes of growth whose features are quite different. The films whose initial thickness $h_{i}$ is larger than $h^{*}$ will be considered as "thick" films, while the others $\left(h_{i} \ll h^{*}\right)$ will be the "thin" ones.

\section{RESULTS}

Note first that for a purely viscous liquid $(\Phi(\dot{\gamma} \tau)=\dot{\gamma} \tau)$, our model leads to a constant and uniform thickness for the film, with an exponential growth of the dry radius:

$$
\left\{\begin{array}{l}
R_{d}(t)=R_{0} e^{\frac{|S| t}{\sigma_{0} \tau h_{i}}}=R_{0} e^{\frac{t}{\tau_{i}}} \\
h_{m}(t) \approx h_{i}
\end{array}\right.
$$

This is in complete agreement with the experimental results of Debrégeas et al. for the dewetting of suspended polymer films 12 and supported films [32].

How are affected the growth laws of the dry radius $R_{d}(t)$ and the rim height $h_{m}(t)$ by the shear-thinning properties of the material ?

In order to discuss the time dependence of the rim height and the dry radius, we need to compare $h_{i}$ with the characteristic thickness $h^{*}$. 


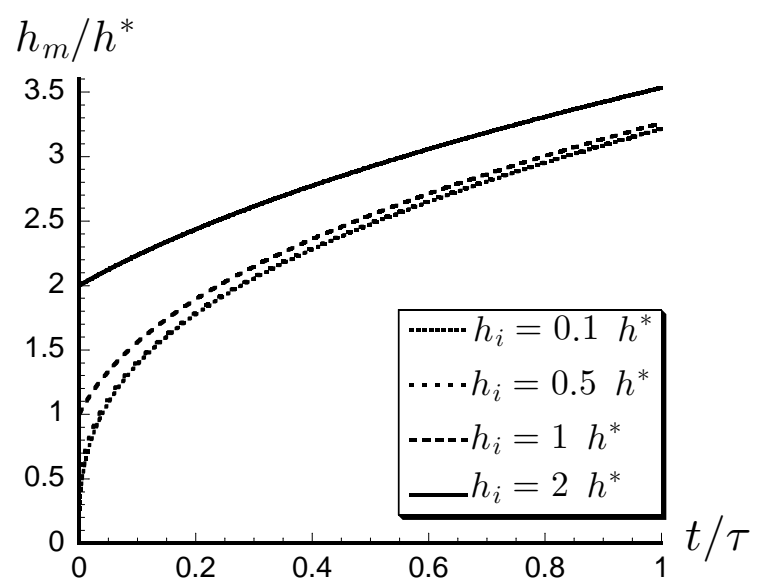

FIG. 4: Short-time evolution of the rim height $h_{m}$ : plot of $h_{m}$ versus $t / \tau$, for $h_{i}=0.1,0.5,1$ and $2 h^{*}$. Note that the curves corresponding to $h_{i}=0.1$ and $0.5 h^{*}$ cannot be distinguished within the graph scale.

\section{A. Thin films regime $\left(h_{i} \ll h^{*}\right)$}

\section{Initial stages of growth}

The range $h_{i} \ll h^{*}$ allows different regimes of growth for the initial stage of hole formation, depending on the specific characteristics of the constitutive law.

Simple analytical laws can be obtained with the Cross law $\left(\Phi(\dot{\gamma} \tau)=(\dot{\gamma} \tau) /\left(1+k(\dot{\gamma} \tau)^{n}\right)\right)$. It can be shown that the solution $\alpha[\beta]$ of Eq.9 is characterized by $\alpha \sim \beta$ for low strain-rates, and $\alpha \sim \psi(n) \beta$ for $\beta \gg 1$. It is worth noting that the coefficient $\psi(n)$ does not depend on $k$, but depends on $n$ as a solution of:

$$
\left(\psi^{1-n}+(\psi-1)^{1-n}\right)(\psi+1)(1-n)=\psi^{1-n}+1
$$

As shown in Fig.3, this coefficient ranges from $\psi=1$ (for $n \rightarrow 0$ ) to $\psi \rightarrow+\infty$ (for $n \rightarrow 1$ ), and is involved in the laws of growth relative to the rim height and dry radius, as shown below.

The strain-rates $\alpha_{m}(t)$ and $\beta_{m}(t)$ at the rim edge (i.e. for $\left.r=R_{d}(t)\right)$ are simply related to the rim height by balancing the radial stress acting on the rim and the capillary forces. This gives the following equation:

$$
\frac{h^{*}}{h_{m}}=\frac{\alpha_{m}}{1+k \alpha_{m}^{n}}+\frac{\alpha_{m}-\beta_{m}}{1+k\left(\alpha_{m}-\beta_{m}\right)^{n}},
$$

admitting the approached forms $\beta_{m} \sim h^{*} / h_{m}$ for large values of $h_{m} / h^{*}$, and $\beta_{m} \sim \mu(n, k)\left(h^{*} / h_{m}\right)^{1 / 1-n}$ for the thin films regime. $\mu(n, k)$ is a positive dimensionless parameter depending on $n$ and $k$ as:

$$
\mu(n, k) \equiv \frac{k}{\psi(n)^{1-n}+(\psi(n)-1)^{1-n}}
$$

These expressions of strain-rates at the edge of the rim versus the rim height enable us to find analytical expressions of the growth laws during the initial stages of dewetting for thin films.
The rim height obeys the equation:

$$
\frac{1}{h_{m}} \frac{\mathrm{D} h_{m}}{\mathrm{D} t}=\frac{\alpha_{m}-\beta_{m}}{\tau},
$$

where $\mathrm{D} \equiv \frac{\partial}{\partial t}+v \frac{\partial}{\partial r}$ stands for the particular derivative. In Fig. 4 we present our results about the short-time evolution of rim height for different initial thicknesses, with $t$ ranging from 0 to $\tau$. The solution of Eq.15 along with the initial condition $h_{m}(t=0)=h_{i}$ is:

$$
h_{m}(t)=h_{i}\left(1+\lambda\left(n, k, h_{i}\right) \frac{t}{\tau}\right)^{1-n}
$$

with $\lambda\left(n, k, h_{i}\right) \equiv k \frac{\psi^{2}-1}{\psi^{1-n}+1}\left(\frac{h^{*}}{h_{i}}\right)^{1 / 1-n}$. The adequation between our numerical results and this theoretical prediction is excellent.

The dry radius is given by:

$$
\frac{\dot{R}_{d}}{R_{d}}=\frac{\beta_{m}(t)}{\tau}
$$

and we thus obtain:

$$
R_{d}(t)=R_{0}\left(1+\lambda\left(n, k, h_{i}\right) \frac{t}{\tau}\right)^{\frac{1-n}{\psi-1}}
$$

It is physically understandable that $R_{d}$, at a given time $t$, is larger for thinner films : for a given applied force $|S|$ per unit length, the thicker the film, the more the material to be displaced and the lower the dewetting velocity.

Equations 16,18 allow one to define a crossover time $t_{c}=\tau / \lambda\left(n, k, h_{i}\right)$. At short times $\left(i . e . t \ll t_{c}\right), h_{m}$ and $R_{d}$ vary linearly with time: $h_{m} \approx h_{i}\left(1+(1-n) t / t_{c}\right)$ and $R_{d} \approx R_{0}\left(1+\frac{(1-n)}{\psi-1} t / t_{c}\right)$. At longer times $\left(t \gg t_{c}\right)$, the time dependence of $h_{m}$ and $R_{d}$ is a power-law: $h_{m} \sim t^{1-n}$ and $R_{d} \sim t^{(1-n) /(\psi-1)}$. Note that the thinner the film, the smaller the crossover time $t_{c}$.

A simple expression of $R_{d}$ versus $h_{m}$ can be directly derived from Eqs.16 and 18:

$$
R_{d}(t)=R_{0}\left(\frac{h_{m}(t)}{h_{i}}\right)^{\frac{1}{\psi-1}}
$$

This relationship between the dry radius and the rim height is shown in Fig for different values of power-law index $n$. Note that, for $n=2 / 3$, the expression (19) turns out to be linear: this important property will be discussed in the light of Reiter's experiments in part IV B.

\section{Long-time regime of growth}

After the initial stages of hole growth, the driving force of the phenomenon, $|S|$, is distributed over a rim height larger than the characteristic thickness $h^{*}$. The resulting radial constraint becomes weak, and gives rise to very small values of strain-rates $\alpha$ and $\beta$. Then, to solve Eq.15 


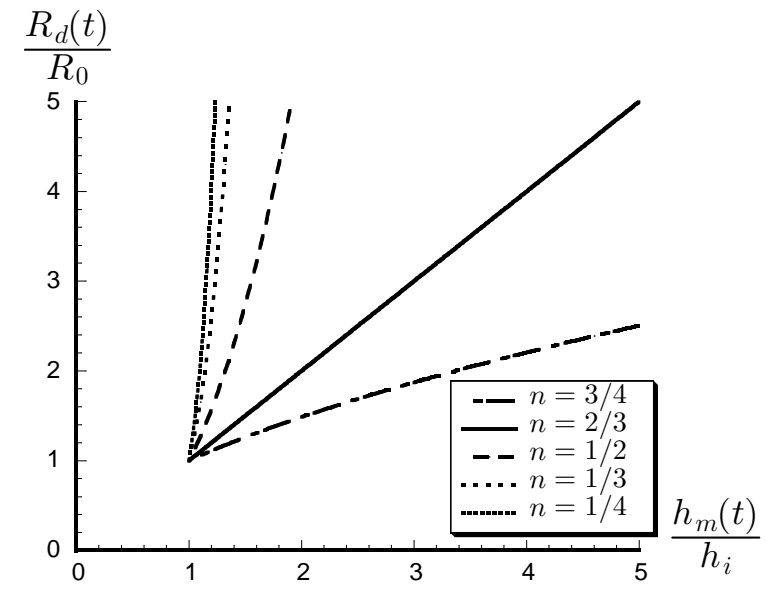

FIG. 5: For thin films $\left(h_{i} \ll h^{*}\right)$, during the initial stages of growth, the dry radius is related to the rim height by a simple power-law depending on the material rheology (Eq.19). This figure shows the different regimes depending on $n$ : for $n=2 / 3$, the dependence is simply linear; for $n>2 / 3$, the radius grows slower than the rim height, while for $n<2 / 3$, $R_{d}$ grows fastly compared to the rim height increasing.

in this regime, we have to find the smallest-order correction to the linear behavior of function $F$ :

$$
\alpha=F(\beta) \underset{\beta \ll 1}{\approx} \beta+\epsilon(\beta)
$$

Including this development in Eq.9, and neglecting terms involving second-order (or higher) $\epsilon$, we obtain a nonlinear first-order differential equation for $\epsilon$. Solving it along with the condition $\epsilon(\beta=0)=0$ leads to an explicit expression for $\epsilon(\beta)$. Keeping the smallest order in development of $\epsilon$ for small $\beta$, we find [33]:

$$
\alpha \underset{\beta \ll 1}{\approx} \beta+\left(\frac{n}{n+1} k\right) \beta^{n+1}
$$

From Eq.15, it is then easy to obtain the following expression for $h_{m}$ :

$$
h_{m}(t)=h_{i}\left(1+\nu\left(n, k, h_{i}\right) \frac{t}{\tau}\right)^{\frac{1}{1+n}}
$$

with $\nu\left(n, k, h_{i}\right) \equiv n k\left(\frac{h^{*}}{h_{i}}\right)^{1+n}$. We have checked numerically (cf. Fig.6) that this power-law behavior is obeyed in the long-time regime of growth.

The corresponding law for dry radius in this regime is:

$R_{d}(t)=R_{0} \exp \left[\frac{1+n}{n k^{2}}\left(\frac{h_{i}}{h^{*}}\right)^{n}\left(\left(1+\nu\left(n, k, h_{i}\right) \frac{t}{\tau}\right)^{\frac{n}{1+n}}-1\right)\right]$

For $t \gg \tau\left(h_{i} / h^{*}\right)^{1+n} /(n k)$, this law turns out to be a stretched exponential:

$$
R_{d}(t) \approx R_{0} e^{\frac{1+n}{k}(n k)^{-\frac{1}{1+n}}\left(\frac{t}{\tau}\right)^{\frac{n}{1+n}}}
$$

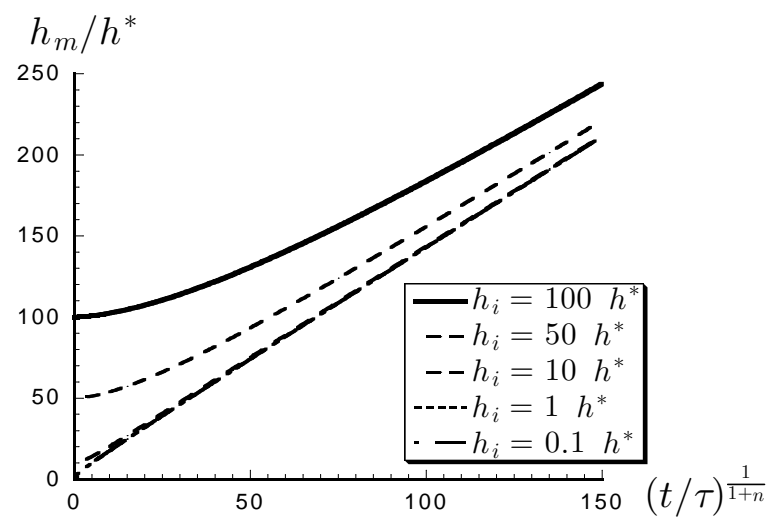

FIG. 6: Long-time evolution of the rim height $h_{m}$, for different initial thicknesses $h_{i}\left(0.1 ; 1 ; 10 ; 50 ; 100 h^{*}\right)$, with Cross law $(n=2 / 3, k=5)$ : plot versus $(t / \tau)^{\frac{1}{1+n}}$ to show the linear behavior of $h_{m}$ with $(t / \tau)^{\frac{1}{1+n}}$ after the transient time $t_{0}$. For a thick film $\left(h_{i} \gg h^{*}\right)$, two regimes can be distinguished. For $t<t_{0} \sim \tau\left(\frac{h_{i}}{h^{*}}\right)^{1+n}, h_{m}$ is constant and $R_{d}(t)$ increases exponentially with time. For $t>t_{0}, h_{m}$ growths linearly with $(t / \tau)^{\frac{1}{1+n}}$. Note that the curves corresponding to $h_{i}=0.1$ and $1 h^{*}$ cannot be distinguished within the graph scale.

From Eq.16, it is easy to define a crossover time $t_{e}$ between the initial stages (part III A 1) and the long-time regime of growth (part III A 2):

$$
t_{e}=\mu\left(\frac{h^{*}}{h_{i}}\right)^{\frac{1}{1-n}} t_{c}
$$

\section{Film profile}

An example of profile obtained for $h_{i}=0.1 h^{*}$ is shown in Fig.7. This profile is characterized by a highly asymmetric shape for the rim. There is a striking similarity between such a profile and those observed by Reiter for ultra-thin films [17]. On fig.8 we present the time evolution of this profile (for $t$ ranging from $t=0$ to $t=5.10^{-2} \tau$, with a time step of $10^{-2} \tau$ ).

For the sake of conciseness, we focus there on the case corresponding to the range of thicknesses and times covered by Reiter's experiments, i.e. the initial times of growth for thin films (cf. 17] and IV). For clarity reasons, let us write $\Omega(t) \equiv 1+\lambda\left(n, k, h_{i}\right) \frac{t}{\tau}$. In the regime $h_{i} \ll h^{*}$, the partial derivative equation 5 for $h(r, t)$ reduces to:

$$
\frac{\partial h(r, t)}{\partial t}+\frac{\kappa}{r^{\psi}} \Omega(t)^{\frac{2-n-n \Psi}{\psi-1}} \frac{\partial h}{\partial r}=(\psi-1) \frac{\kappa}{r^{1+\psi}} \Omega(t)^{\frac{2-n-n \Psi}{\psi-1}} h
$$

The resolution of Eq.26 can be achieved using a method of characteristics, as explained in appendix $\mathbb{D}$. 


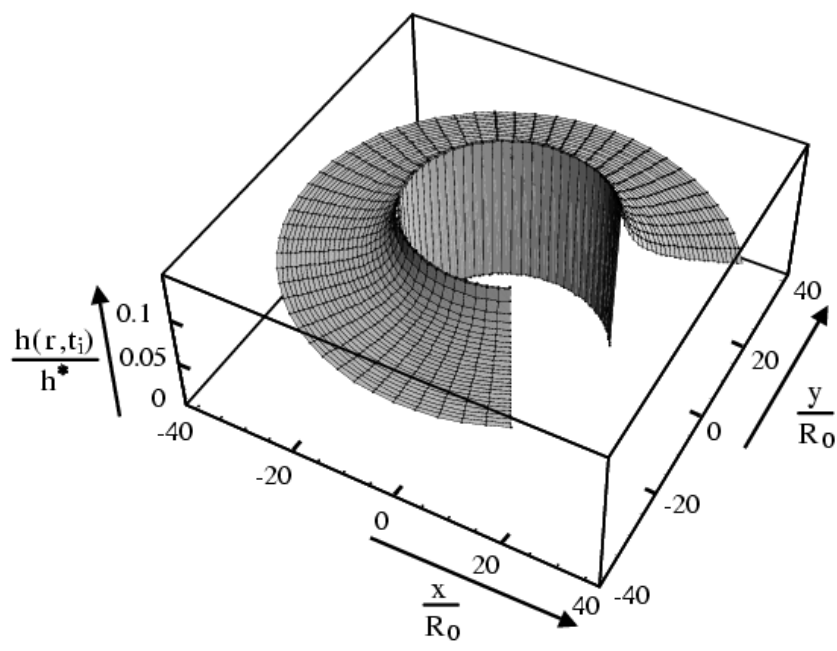

FIG. 7: Film profile for $h_{i}=0.1 h^{*}$ and $t=2 \cdot 10^{-2} \tau$. The constitutive equation of the material is the Cross law (Eq.4), with a power-law index $n=2 / 3$. For clarity reasons, a sectional view is displayed on a quarter of circle, and cartesian coordinates $(x, y, z)$, naturally associated with cylindrical ones $(r, \Phi, z)$, are used.

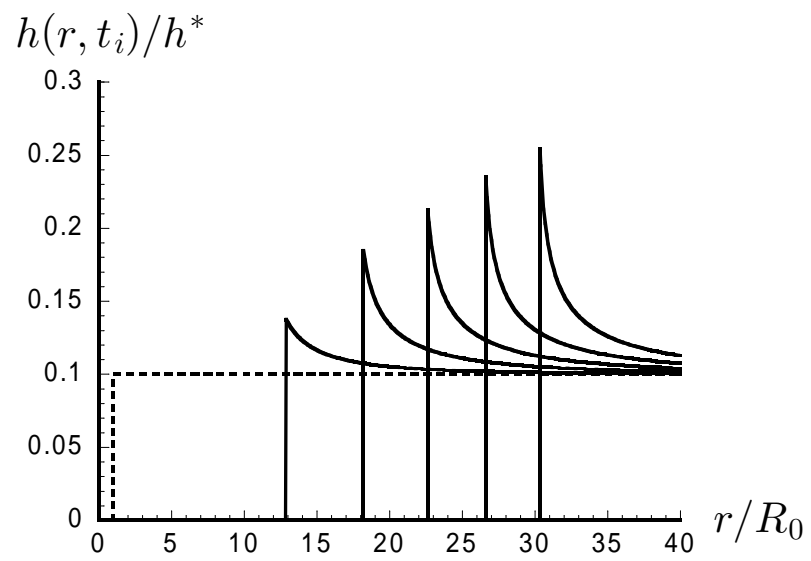

FIG. 8: Evolution of a film (Cross model, $n=2 / 3$ ) profile $h\left(r, t_{i}\right)$, for $h_{i}=0.1 h^{*}$ and $t_{i}$ ranging from $t=0$ (initial configuration, in dashed lines) to $t=5 \cdot 10^{-2} \tau$ with a time step of $10^{-2} \tau$.

The solution is:

$$
h(r, t)=h_{i} \frac{r^{\psi-1}}{\left(1+r^{\psi+1}-R_{d}(t)^{\psi+1}\right)^{\frac{\psi-1}{\psi+1}}}
$$

We checked (cf. appendix D) that this expression has a wide range of validity: during all the initial growth, the strain-rates remain high ( $\alpha$ and $\beta \gg 1$ ) far away the hole periphery, and thus Eq.27 is an acceptable description of the whole film profile.

\section{B. Thick films regime $\left(h_{i} \gg h^{*}\right)$}

For thick films, as shown in Fig.6 (for instance in the case $h_{i}=100 h^{*}$ ) two different time regimes can be distinguished: at the beginning of hole formation, $h_{m}$ is nearly constant and equals its initial value $h_{i}$ during a long period of time, before growing faster. Here again, a distinction between different time regimes is necessary.

\section{Initial stages of growth}

During the early stage, the strain rates at the rim are constant and small, as a consequence of the constant large thickness. In this case, the rheological law of the film is viscous-type and the dry radius increases exponentially, as for the case previously discussed (see Eq.11):

$$
\left\{\begin{array}{l}
R_{d}(t)=R_{0} e^{\frac{|S| t \mid t}{\sigma_{0} \tau h_{i}}}=R_{0} e^{\frac{t}{\tau_{i}}} \\
h_{m}(t) \approx h_{i}
\end{array}\right.
$$

This viscous-type behavior remains valid up to a crossover time $t_{0} \approx \tau\left(\frac{h_{i}}{h^{*}}\right)^{1+n}$.

\section{Long-time regime of growth}

We have checked numerically (cf. Fig.6) that the anticipated analytical behavior

$$
h_{m}(t)=h_{i}\left(1+\nu\left(n, k, h_{i}\right) \frac{t}{\tau}\right)^{\frac{1}{1+n}}
$$

is obeyed after the crossover time $t_{0}$.

Similarly, the preceding exponential law for the dry radius connects with the stretched exponential, whose power depends on power-law index $n$ (cf. Eq.24):

$$
R_{d}(t) \approx R_{0} e^{\frac{1+n}{k}(n k)^{-1 / 1+n}\left(\frac{t}{\tau}\right)^{\frac{n}{1+n}}}
$$

Fig. 9 which present the time evolution of $R_{d}$ for different initial thicknesses $h_{i}$ with the Cross model, shows that this anticipated behavior is obeyed for $t \gg t_{0}$.

We emphasize the fact that the crossover from a simple exponential to the stretched exponential regime is a consequence of the non-linearity of rheological law 1 .

\section{DISCUSSION}

\section{A. Debregeas' experiments}

G. Debrégeas et al. [12] carried out experiments on viscous bursting of freely suspended films of long-chain polymers. The high molecular weights of polydimethylsiloxane used in these experiments lead to high viscosities 


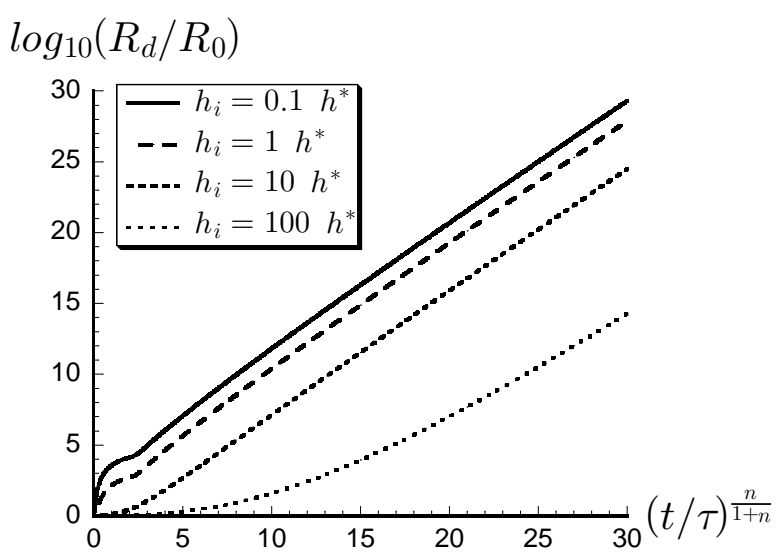

FIG. 9: Logarithmic plot of the dry zone radius $R_{d}$ versus $(t / \tau)^{n /(1+n)}$, for $h_{i}=0.1,1,10$ and $100 h^{*}$, in the case of Cross law with $n=2 / 3$ and $k=5$. Note the linear behavior of $\log _{10}\left[R_{d}(t) / R_{0}\right]$ with $(t / \tau)^{n /(1+n)}$ after a transient time: the anticipated stretched exponential (Eq,24) is indeed obeyed.

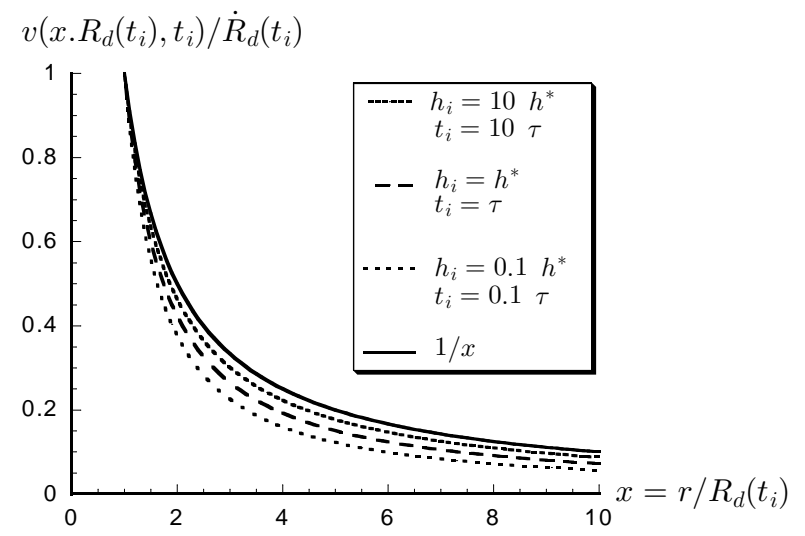

FIG. 10: Velocity field $v\left(r, t_{i}\right)$ for different initial thicknesses $\left(h_{i}=0.1 ; 1 ; 10 h^{*}\right)$, at a given time $t_{i}$. All radial distances are normalized by the dry radius at time $t_{i}$ for each thickness. A curve $1 / x$ is represented for comparison.It is clear that the velocity field is almost proportional to $1 / r$ for a thick profile $\left(h_{i}=10 h^{*}\right)$, similar to the long range radial plug flow observed by Debrégeas.

$\left(\eta_{0} \geq 600000 \mathrm{cP}\right)$ bursting processes where viscous dissipation dominates inertia.

The range of thicknesses experimentally studied by Debrégeas et al. (5 to $250 \mu \mathrm{m})$ covers the domain $h_{i} \gg h^{*}$. As the corresponding crossover time is very large $\left(t_{0} \gg \tau\right)$, these experiments mainly covered the first regime $t \ll t_{0}$ (the criterion $h_{i} \gg h^{*}$ is in fact a basic hypothesis of their "soft balloon" model [34). In our model, the velocity field $v(r, t)$ is found to be almost proportional to $1 / r$ at any time for thick profiles (cf. Fig 10), similar to the experimentally observed long range radial plug flow [12].

Thus, our model accounts well for the exponential growth and the absence of rim characteristics of the dewetting regime observed by Debrégeas et al. for vis- cous polymer films.

\section{B. Reiter's experiments}

G. Reiter [17 studied the dewetting of ultra-thin, almost glassy polystyrene films deposited onto silicon wafers coated with a polydimethylsiloxane monolayer. The thicknesses of the PS films used range from $h_{i}=10$ to $h_{i}=60 \mathrm{~nm}$. Compared with the characteristic scale $h^{*} \approx 50 \mathrm{~nm}$, the ratio $h_{i} / h^{*}$ ranges from about 0.2 to unity. The characteristic time $\tau$ for PS under the experimental conditions is very long, up to more than a year for the lowest temperatures conditions $\left(T\right.$ ranges from $103^{\circ} \mathrm{C}$ to $130^{\circ} \mathrm{C}$, to be compared with the bulk glass transition temperature: $T_{g}=97.5^{\circ} \mathrm{C}$ ). We can thus expect that the relevant regime for comparing our results with Reiter's experimental findings is the limit $h_{i} \ll h^{*}$, and $t / \tau$ small (cf. III A 1).

Three years ago, Dalnoki-Veress et al. made an experimental study of hole formation and growth in freely standing PS films [22]. In agreement with Debregeas' experiments, they observed exponential growth of the hole radius and uniform thickening, but with a deviance from this regime for long-time regimes of growth, a feature they analyzed in terms of shear-thinning properties. They indeed characterized the polymer rheology at the temperature of their experiments $\left(T=115^{\circ} \mathrm{C}\right)$ and found that the best fit for the viscosity $\eta$ versus the shear strain rate $\dot{\gamma}$ is a power-law dependence:

$$
\eta \sim|\dot{\gamma}|^{-d},
$$

with a power-law index $d=0.65 \pm 0.03$. For the following, let us suppose that the rheology of ultra-thin PS films used by Reiter can be described by the Cross model (Eq.1) with such a power-law index: $n=2 / 3$. It is of course an hypothesis that would need an experimental validation. In particular, the rheology of PS films at temperatures very close to the glass transition may be quite different. Keeping this point in mind, the following remarks are tentative explanations of some regimes of dewetting experimentally observed by Reiter.

If $n=2 / 3$, Eq.12 leads to the exact value $\psi=2$. Then Eq.19 turns out to be simply linear:

$$
\frac{R_{d}(t)}{R_{0}}=\frac{h_{m}(t)}{h_{i}}
$$

This property was indeed observed by Reiter: the maximum height of the rim grows at the same speed as the radius of the hole 17. The special role played by this value $n=2 / 3$ was also pointed out by Shenoy and Sharma in their study of the dewetting of power hardening viscoplastic solid 21].

We can also focus on the individual dependences of $h_{m}$ and $R_{d}$ versus time. As precised in part III A 1, for 
$t \gg t_{c}$, the rim height and the hole radius follow a powerlaw time dependence: $h_{m} \sim t^{1-n}$ and $R_{d} \sim t^{(1-n) /(\psi-1)}$. In particular, for $n=2 / 3$, we obtain:

$$
\left\{\begin{array}{l}
h_{m} \sim(t / \tau)^{1 / 3} \\
R_{d} \sim(t / \tau)^{1 / 3}
\end{array}\right.
$$

Very recently, Reiter [35] characterized the time dependences of $R_{d}$ and $h_{m}$ for very thin films. He obtained several dewetting regimes, and work is in progress to analyze these results. It seems that several experimental factors must be taken into account for this analysis. Among them, the influence of film preparation appears to be decisive: the spin-cast PS film is a highly metastable form of matter [5]. The sample preparation (including the time of healing) can lead to thin films where residual constraints on the chains persist, and may have an influence on the dewetting process.

Quite interestingly, despite all these remarks, for a 20 $\mathrm{nm}$ PS film deposited on a $10 \mathrm{~nm}$ PDMS layer around $T=120^{\circ} \mathrm{C}$, the results of Reiter's experiments seem to indicate that the maximum height of rim and the hole radius obey power-laws:

$$
\left\{\begin{array}{l}
h_{m} \sim(t / \tau)^{a} \quad \text { with } a \approx 0.38 \pm 0.02 \\
R_{d} \sim(t / \tau)^{b} \quad \text { with } b \approx 0.34 \pm 0.04
\end{array}\right.
$$

The time range on which Reiter calculated these power-law indexes is up to about 100 minutes. As for a $20 \mathrm{~nm}$ film, the ratio $h_{i} / h^{*}$ is smaller than unity, the crossover time $t_{c}$ is very short, and we can expect that the power-law regime (Eq.32) is established. Thus we can compare Eqs.32 and 33 and remark that the two experimental coefficients are indeed very close to the predicted one: $1 / 3$. This adequation between our model and Reiter's experimental results appears to be satisfying. But many questions remain open, as the role played by the thin PDMS monolayer on which the PS film dewets is only taken into account by the free slippage condition [36]. In fact, it was shown by several studies that the lower layer is deformed by the moving upper one [37, which can leads to viscous dissipation and to a marked decrease in the dewetting velocity [38].

Let us conclude by a tentative idea: observing by TappingMode atomic force microscopy (TM-AFM) the dry zone on the PDMS substrate after the removal of PS film, Reiter 39] discovered the formation of straight lines, several microns long, starting to appear at hole diameters larger than about $1 \mu \mathrm{m}$. These lines are PS microfibrils, whose width can be as low as about $2 \mathrm{~nm}$ (to be compared with the radius of gyration of the polymer in the bulk, of order $50 \mathrm{~nm}$ ). This observation suggests that the radial strain-rates are much larger than the orthoradial ones at the hole periphery and involve this elongational deformation of the molecules. This property could be related to the fact that, in our model, the radial strain-rate $(\alpha)$ is large compared with the orthoradial one $(\beta)$ during the initial stages of growth for thin films (cf. Fig.2, regime $\beta \gg 1$ ). In particular, note that this dissymmetry between values of $\alpha$ and $\beta$ is a direct consequence of the shear-thinning behavior of the polymer: for a purely viscous fluid, $\alpha=\beta$ for any thickness.

\section{CONCLUSION}

In conclusion, our model accounts well for the exponential growth and the absence of rim characteristics of the dewetting regime observed by Debrégeas et al. for viscous polymer films. Taking into account the shearthinning behavior of the polymers near $T_{g}$ enables us to see the modifications induced by this particular rheology on the film morphology. It appears from our results that the early stages of dewetting for a shear-thinning polymer film are mainly determined by the ratio of its initial thickness $h_{i}$ to a characteristic scale $h^{*}$ (related to the driving force of the process, $S$, and the rheological response of the material, characterized by $\sigma_{0}$ ).

The film profile exhibits a sharp, asymmetric rim similar to the one observed by Reiter in his latest experiments with ultrathin PS films. The rich variety of growth behavior is summarized in appendix Q. In all cases, the long-time evolution of $h_{m}$ is a power-law (whose coefficient depends on the rheology of the material studied), and $R_{d}$ obeys a stretched exponential law. When taking for the power-law index $n$ the value obtained by DalnokiVeress et al., in the thin-film limit, our predictions for the dry radius and the rim height time-evolutions seem to explain some of Reiter's latest experiments. In particular, the linear dependence of the rim height versus the dry radius experimentally observed is predicted by our model for the power-law index $n=2 / 3$.

Work is now in progress to incorporate in our model the Laplace pressure (see 28]) which might lead to oscillatory dewetting fronts [14, 18], and to evaluate the possible effects of chain entanglements. In the future, we aim to study the dewetting of thin polymer films below $T_{g}$, where the existence of a yield stress in the rheological response of the material may lead to new dewetting morphologies.

\section{Acknowledgments}

We are grateful to G. Reiter for very useful discussions and for providing us with his experimental results on the dewetting of ultra-thin polystyrene films prior to publication.

We also wish to acknowledge fruitful discussions with A. Aradian, G. Debrégeas, and M. Sferrazza.

\section{APPENDIX A: A FEW REMARKS ABOUT THE VELOCITY FIELD INSIDE THE FILM}

Our choice of model (see III) for the dewetting film deserves a few comments. As defined above, $v(r, t)$ is 
the radial part of flow field. The incompressibility of the material implies the additional existence of a small, but non-zero vertical component of the velocity field: let us note $w$ this vertical part.

We already argued that the characteristic thickness of films studied by Reiter and Debrégeas allows a plug-flow assumption for the film: it implies that the radial part $v(r, t)$ is independent of variable $z$. This hypothesis does not imply the same property for $w$ : we must keep for it the most general dependence $w(r, z, t)$.

In cylindrical coordinates, for a velocity field of the form $\left(v_{r}, v_{\Phi}, v_{z}\right)$, the liquid incompressibility leads to:

$$
\frac{\partial v_{r}}{\partial r}+\frac{v_{r}}{r}+\frac{1}{r} \frac{\partial v_{\Phi}}{\partial \Phi}+\frac{\partial v_{z}}{\partial z}=0
$$

Here, as $v_{\Phi}=0$ for symmetry reasons, one has:

$$
\frac{\partial v}{\partial r}+\frac{v}{r}=-\frac{\partial w}{\partial z}
$$

Independence of $v$ versus $z$ allows a simple integration of EqA2 at a given $r$ :

$$
\int_{z=0}^{h(r, t)}\left(\frac{\partial v}{\partial r}+\frac{v}{r}\right) \mathrm{d} z=-\int_{z=0}^{h(r, t)} \frac{\partial w}{\partial z} \mathrm{~d} z
$$

which gives:

$$
h(r, t) \cdot\left(\frac{\partial v}{\partial r}+\frac{v}{r}\right)=-w(r, z=h(r, t), t)
$$

The vertical velocity at the top of the film, $w(r, z=$ $h(r, t), t)$, can be easily related to the particular derivative of $h(r, t)$, provided that the film thickness remains small compared with the characteristic lengths of variation of the radial speed component and film thickness 40]:

$$
w(r, z=h(r, t), t) \approx\left(\frac{\partial h}{\partial t}+v \cdot \frac{\partial h}{\partial r}\right)
$$

Thus, in a more rigorous way, we recover the incompressibility condition (see Eq.5):

$$
\frac{1}{h(r, t)}\left(\frac{\partial h(r, t)}{\partial t}+\frac{r \beta(r, t)}{\tau} \frac{\partial h(r, t)}{\partial r}\right)=\frac{\alpha(r, t)-\beta(r, t)}{\tau}
$$

The plug-flow assumption has a direct consequence: the left-hand side of Eq. A2 is independent of $z$; so does the right-hand side, i.e. $\partial w / \partial z$, which implies that $w$ has a simple linear dependence versus $z$ :

$$
w(r, z, t)=w(r, z=h(r, t), t) \frac{z}{h(r, t)}
$$

To finish with this point, note that taking into account this small but non-zero vertical component of the velocity field induces the presence of a supplementary term in the conservation of momentum (equation 8):

$$
\frac{\partial \sigma_{r r}}{\partial r}+\frac{\partial \sigma_{r z}}{\partial z}+\frac{\sigma_{r r}-\sigma_{\phi \phi}}{r}=0
$$

The possible consequences of this supplementary friction force on our predictions for dewetting regimes will be studied in a forthcoming publication.

\section{APPENDIX B: RESULTS FOR LOGARITHMIC FORM OF $\Phi$}

For polymers, just above $T_{g}$, it is expected within the framework of the free-volume model that $\sigma^{m}$ varies logarithmically with $\dot{\gamma}$ as 24]:

$$
\Phi(\dot{\gamma} \tau)=\ln (1+\dot{\gamma} \tau)
$$

At low strain-rates $(\dot{\gamma} \tau<1)$, this law displays a viscous-type behavior $\left(\sigma^{m} \approx \eta_{0} \dot{\gamma}\right.$, with a zero-shear viscosity $\eta_{0}=\sigma_{0} \tau$ ), while for large values of $\dot{\gamma} \tau, \sigma^{m}$ reaches an almost constant value (shear-thinning behavior).

In order to deal with a minimum number of parameters, we assume that $\sigma^{m}(\dot{\gamma})$ is an odd function $\left(\sigma^{m}(\dot{\gamma})=\right.$ $-\sigma_{0} \ln (1+|\dot{\gamma}| \tau)$ if $\left.\dot{\gamma}<0\right)$.

This appendix briefly presents the main results for thin and thick films.

For thick films, at short times, the dry radius and rim height evolve as for a purely viscous fluid (cf. Eq.11):

$$
\left\{\begin{array}{l}
R_{d}(t)=R_{0} e^{\frac{|S| t}{\sigma_{0} \tau h_{i}}}=R_{0} e^{\frac{t}{\tau_{i}}} \\
h_{m}(t) \approx h_{i}
\end{array}\right.
$$

For thick films, at long times $\left(t>t_{0} \sim \tau\left(h_{i} / h^{*}\right)^{2}\right)$, the rim height grows proportionally to $\sqrt{t / \tau}$ :

$$
h_{m}(t) \underset{t \gg t_{0}}{\sim} \frac{h^{*}}{2} \sqrt{t / \tau}
$$

while the dry radius expands like $\exp (4 \sqrt{t / \tau})$ :

$$
R_{d}(t) \underset{t \gg t_{0}}{\sim} R_{\infty} e^{4 \sqrt{t / \tau}} .
$$

This law is also a stretched exponential, as for the Cross law. This property is due to the fact that the function $\alpha=F(\beta)$ is analytical (in the mathematical sense), i.e. admits a series expansion near 0 whose first non-linear term is $\beta^{2}$. It indeed corresponds the limit behavior obtained by taking the Cross law with $n \rightarrow 1$.

For thin films, at short times, a sharp rim grows very quickly (with an initial speed $\dot{h}_{m} \approx h_{i} \exp h^{*} / 2 h_{i}$ ). In 
this regime, $h_{m}(t)$ is well described by the integral equation:

$$
\int_{\frac{h^{*}}{2 h_{m}(t)}}^{\frac{h^{*}}{2 h_{i}}} \frac{e^{-x}}{x} \mathrm{~d} x \underset{t \ll \tau}{\approx} \frac{t}{\tau}
$$

For thin films, at long times, as for the Cross law, the hole growth connects with the long-time regime of thick films (Eqs.B2. B3).

\section{APPENDIX C: SUMMARY OF THE DEWETTING REGIMES FOR A CROSS CONSTITUTIVE EQUATION}

Figures 11 and 12 propose a summary of the different dewetting time regimes for a Cross constitutive law, depending on the initial thickness (to be compared with $h^{*}$ ), and relative to different time ranges (to be compared with crossover times $t_{c}, t_{e}$ and $t_{0}$ ).

\section{APPENDIX D: ANALYSIS OF THE FILM PROFILE}

\section{Method of characteristics}

To know the profile $h(r, t)$, the equation 26 has to be solved:

$\frac{\partial h(r, t)}{\partial t}+\frac{\kappa}{r^{\psi}} \Omega(t)^{\frac{2-n-n \Psi}{\psi-1}} \frac{\partial h}{\partial r}=(\psi-1) \frac{\kappa}{r^{1+\psi}} \Omega(t)^{\frac{2-n-n \Psi}{\psi-1}} h$

along with the precedently precised initial condition (Eq.10) and the boundary values $h(r \rightarrow \infty, t)=h_{i}(\forall t)$ and $h\left(r=R_{d}(t), t\right)=h_{m}(t)$. As a a quasi-linear partial derivative equation, Eq.26 can be solved using a method of characteristics, as precised in this appendix.

Writing $\mathrm{d} h(r, t)=\frac{\partial h}{\partial t} \mathrm{~d} t+\frac{\partial h(r, t)}{\partial r} \mathrm{~d} r$, we can replace the partial derivative with respect to $t$ thanks to Eq26. Let define a characteristic by the function $r_{\xi}(t)$, indexed by the parameter $\xi$ with $r_{\xi}(t=0)=\xi$, and obeying the following ordinary differential equation:

$$
\frac{\mathrm{d} r_{\xi}(t)}{\mathrm{d} t}=\frac{\kappa}{r_{\xi}(t)^{\psi}} \Omega(t)^{\frac{2-n-n \psi}{\psi-1}}
$$

Eq.D1 is tractable, and gives each characteristic as a function of time:

$$
r_{\xi}(t)^{\psi+1}-\xi^{\psi+1}=\Omega(t)^{\frac{(\psi+1)(1-n)}{\psi-1}}-1
$$

In Fig.13 is shown a representation of characteristics $r_{\xi}(t)$ versus time, for a thin film $\left(h_{i}=0.1 h^{*}\right)$. Note that these curves were extracted numerically from our model, and are not a graphic illustration of solutions of Eq.D2.
Along a given characteristic, the rim height $h_{\xi}(t)$ obeys a simple equation:

$$
\frac{\mathrm{d} h_{\xi}(t)}{\mathrm{d} t}=\frac{\kappa(\psi-1)}{r_{\xi}(t)^{\psi+1}} \Omega(t)^{\frac{(\psi+1)(1-n)}{\psi-1}} h_{\xi}(t),
$$

whose solution is explicit and of the form:

$$
h_{\xi}(t)=h_{i}\left(\frac{\Omega(t)^{\frac{(\psi+1)(1-n)}{\psi-1}}+\xi^{1+\psi}-1}{\xi^{1+\psi}}\right)^{\frac{\psi-1}{\psi+1}}
$$

Inverting Eq.D2 to express $\xi$ as a function of $r$ and $t$ enables us to extract the complete equation of the profile (Eq.27):

$$
h(r, t)=h_{i} \frac{r^{\psi-1}}{\left(1+r^{\psi+1}-R_{d}(t)^{\psi+1}\right)^{\frac{\psi-1}{\psi+1}}}
$$

\section{Range of validity}

In the film zone near the hole $\left(r \gtrsim R_{d}(t)\right)$, the strainrates $\alpha$ and $\beta$ are high. Thus Eq. 7 gives:

$$
\beta(r, t)=\beta_{m}(t)\left(\frac{R_{d}(t)}{r}\right)^{1+\psi}=\kappa \frac{\Omega(t)^{\frac{2-n-n \psi}{\psi-1}}}{r^{1+\psi}}
$$

Our description of film profile remains valid as long as $\beta(r, t) \geq 1$. Let us define the radial distance $r^{*}(t)$ up to which this condition is verified : $\beta\left(r^{*}(t), t\right)=1$. The ratio $r^{*}(t) / R_{d}(t)$ is given by:

$$
\frac{r^{*}(t)}{R_{d}(t)}=\kappa \Omega(t)^{\frac{1-n \psi}{\psi-1}}
$$

We regard the equation of profile as valid if $r^{*}(t) \gg$ $R_{d}(t)$, and arbitrarily choose a criterion $r^{*}(t) \geq \Upsilon R_{d}(t)$ with a factor $\Upsilon \sim 10$ for instance. It is then found that $r^{*}(t) \geq \Upsilon R_{d}(t)$ as long as time $t$ is smaller than a critical value $t^{*}$ given by:

$$
t^{*}=\frac{1-n}{\psi-1} \frac{\mu(n, k)^{\frac{\psi(1-n)}{n \psi-1}}}{\Upsilon^{\frac{\psi-1}{n \psi-1}}}\left(\frac{h^{*}}{h_{i}}\right)^{\frac{\psi}{n \psi-1}}
$$

For $h_{i}=0.1 h^{*}, n=2 / 3, k=5$, and $\Upsilon=10$, Eq.D7 shows that the high strain-rates hypothesis remains valid on a wide region as long as $t \lesssim 1000 \tau$. Thus, the Eq.27 is a good description of the film profile during all the initial regime of growth.

\section{APPENDIX E: INFLUENCE OF THE INITIAL SHAPE OF FILM PROFILE}

The results presented above are obtained taking a discontinuous shape for the initial profile of the film:

$$
h(r, t=0)= \begin{cases}h_{i} & \text { if } r \geq R_{0}=1 \\ 0 & \text { otherwise }\end{cases}
$$




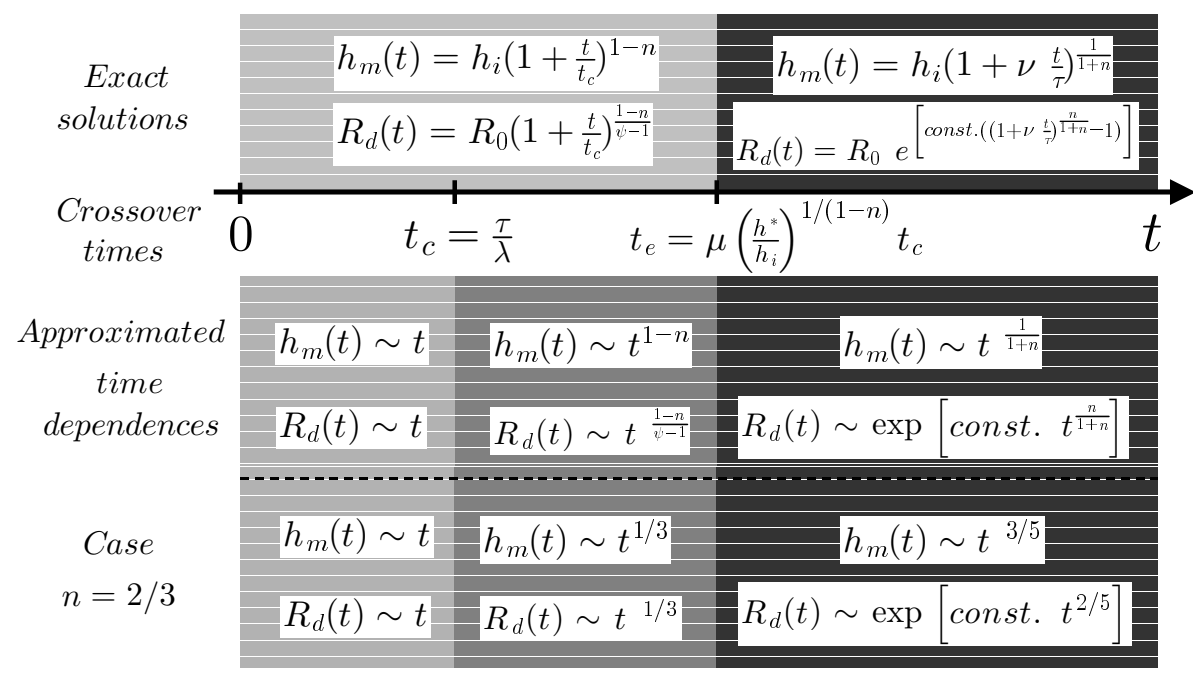

FIG. 11: Summary of the different time regimes for the dewetting of thin films $\left(h_{i} \ll h^{*}\right)$. Depending on each time range, the exact solutions and corresponding approximated time-dependences are precised. We also point out the laws for the special value $n=2 / 3$ (discussed in part IVB)

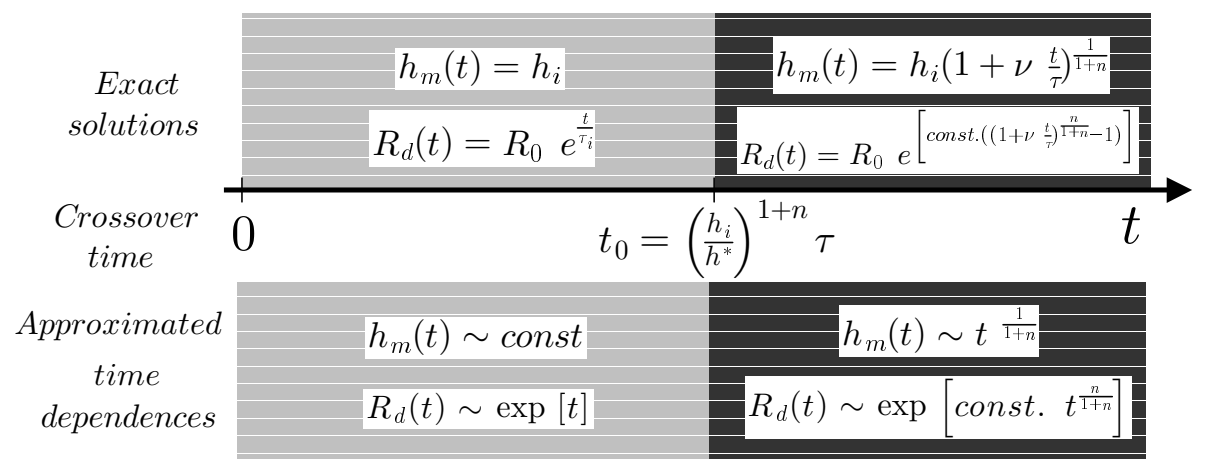

FIG. 12: Summary of the different time regimes for the dewetting of thick films $\left(h_{i} \gg h^{*}\right)$. Depending on each time range, the exact solutions and corresponding approximated time-dependences are precised.

Does this discontinuity at $r=R_{0}$ play a role in the hole growth? In other words, is it the source of the marked asymmetry observed for the profiles obtained with our model?

To probe this hypothesis, we made the same resolution of our system of equations, starting with a continuous profile:

$$
h(r, t=0)= \begin{cases}h_{i} e^{\frac{\chi}{\left(r / R_{0}\right)^{2}-1}} & \text { if } r>R_{0}=1 \\ 0 & \text { if } r \leq R_{0}\end{cases}
$$

This profile is infinitely derivable at $r=R_{0}$, the parameter $\chi$ giving an order of the characteristic length of transition between the regions $h=0$ and $h=h_{i}$. The profiles obtained with this initial condition are shown in figure 14, with $\chi=0.1$ (the shape of the initial profile, represented in dashed lines in Fig.14, is very smooth). The rim growth seems to be very fast for a thin film (the time range of curves in Fig. 14 is 0 to $2.10^{-2} \tau$ ). Similarly, the increasingly asymmetric shape of the profile shows that, even when starting with a smooth profile, the resulting morphology of the rim is the same as in Reiter's experiments. These considerations prove that the discontinuity of the initial profile is not a necessary condition for the appearance, growth and stiffening of an asymmetric rim as those observed by Reiter.
[1] A. Oron, S. H. Davis, and S. G. Bankoff, Rev. Mod. Phys. 69, 931 (1997)
[2] See e.g., G. Reiter, Science 282, 888 (1998); 


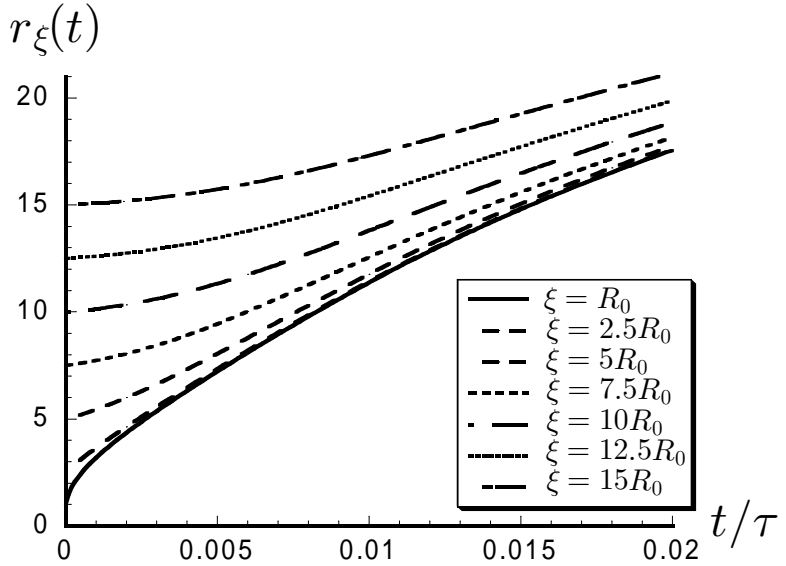

FIG. 13: Graphic representation of characteristics $r_{\xi}(t)$, for an initial thickness $h_{i}=0.1 h^{*}$ and $t$ ranging from 0 to $0.02 \tau$. Different values of $\xi$ parameter are taken: $\xi=1 ; 2.5 ; 5 ; 7.5$; $10 ; 12.5$ and $15 R_{0}$.

FIG. 14: Evolution of the film morphology near the hole periphery (from $R_{d}$ to $R_{d}+R_{0}$ ), starting with a continuous profile (Eq.E2 with $\chi=0.1$, filled with grey color). Each profile, which is relative to the time $t_{i}$ and describes the domain $r \geq R_{d}\left(t_{i}\right)$, is shifted to the point $x=\left(r-R_{d}\left(t_{i}\right)\right) / R_{0}=0$, so as to exhibit clearly its increasingly asymmetric shape.

S. Herminghaus et al., Science 282, 916 (1998)

[3] J.-L. Masson and P. F. Green, Phys. Rev. E 65, 031806 (2002)

[4] J. A. Forrest, K. Dalnoki-Veress, and J. R. Dutcher, Phys. Rev. E 56, 5705 (1997)

[5] G. Reiter, and P.-G. de Gennes, Eur. Phys. J. E. 6, 25 (2001)

[6] G. I. Taylor, Proc. R. Soc. London, Ser. A. 253, 313 (1959)

[7] F. E. C. Culick, J. Appl. Phys. 31, 1128 (1960)

[8] For earlier work on the subject, see: A. Dupré, Ann. Chim. Phys. 11, 194 (1867)
[9] For a discussion on breaking of nonuniform liquid films and threads, based on a simple equation of motion as Taylor and Culick's one, see: J. B. Keller, Phys. Fluids 26, 3451 (1983)

[10] W. R. Mc Entee, and K. J. Mysels, J. Phys. Chem. 73, $3018 / 3028$ (1969)

[11] The precise shape of the rim was later analysed by Keller et al. who showed that it is a cylindrical cap expanding in time like $\sqrt{t}$ : see J. B. Keller, A. King, and L. Ting, Phys. Fluids 7, 226 (1995)

[12] G. Debrégeas, P. Martin, and F. Brochard-Wyart, Phys. Rev. Lett. 75, 3886 (1995);

G. Debrégeas, P.-G. de Gennes, and F. Brochard-Wyart, Science 279, 1704 (1998)

[13] The driving force of the dewetting process is a difference of interfacial tensions: $-S=\gamma_{S L}+\gamma_{L O}-\gamma_{S O}>0$ (where SL corresponds to the solid/liquid interface, SO to solid/air interface, etc...).

[14] Solving numerically the Navier-Stokes equations for long wavelength modulations of the film surface, Brenner and collaborator consider the shape of the rim of a very viscous film retracting under surface tension: see M. P. Brenner and D. Gueyffier, Phys. Fluids 11, 737 (1999)

[15] For molecular simulations on the fluids dynamics of the moving rim, see: J. Koplik, and J. R. Banavar, Phys. Rev. Lett. 84, 4401 (2000)

[16] Kinetics of growth of dry patches (during the early stages of dewetting process of microscopically thin polymer films cast on a solid surface) are simulated by molecular dynamics in: H. Liu, A. Bhattacharya, and A. Chakrabarti, J. Chem. Phys. 109, 8607 (1998)

[17] G. Reiter, Phys. Rev. Lett. 87, 186101 (2001)

[18] For somewhat related studies, see: R. Seemann, S. Herminghaus, and K. Jacobs, Phys. Rev. Lett. 87, 196101 (2001)

[19] F. Saulnier, E. Raphaël, and P.-G. de Gennes, Phys. Rev. Lett. 88, 196101 (2002)

[20] S. Herminghaus, R. Seemann, and K. Jacobs, Phys. Rev. Lett. 89, 056101 (2002)

[21] V. Shenoy and A. Sharma, Phys. Rev. Lett. 88, 236101 (2002)

[22] K. Dalnoki-Veress et al., Phys. Rev. E, 59, 2153 (1999)

[23] P.-G. de Gennes, C. R. Acad. Sci. 288B, 219 (1979)

[24] This Wagner-type constitutive relation can also be obtained from a purely rheological approach, based on the Cox-Merz rule and Eyring's expression for the nonlinear shear viscosity: see J. C. Dyre, Rheol. Acta. 29, 145 (1990)

[25] M. M. Cross, J. Colloid Sci. 20, 417 (1965)

[26] H. A. Barnes, J. F. Hutton, and K. Walters, An introduction to rheology (Elsevier, 1989), p.18

[27] R. M. Christensen, Theory of viscoelasticity, an introduction (Academic Press, 2nd edition, 1982), p.53

[28] In order to keep the model analytically tractable, we here neglect the pressure discontinuity arising from the interface curvature. Taking this Laplace pressure into account may lead to oscillations of film profile, and may even give rise to a cascade of pinch-off events, as proposed in Ref. 20].

[29] C. Redon, F. Brochard-Wyart, and F. Rondelez, Phys. Rev. Lett. 66, 715 (1991)

[30] G. Reiter, Phys. Rev. Lett. 68, 75 (1992)

[31] A theoretical approach to the spinodal dewetting is proposed in: S. A. Safran and J. Klein, J. Phys. II France 
3, 749 (1993)

[32] F. Brochard-Wyart et al., Macromolecules 30, 1211 (1997). This exponential behaviour is observed during the initial stage of process, as long as the viscous friction on the liquid/substrate interface remains negligible compared with the dissipation in the plug flow of removing fluid.

[33] The development of $F(\beta)$ at small $\beta$ shows that $F$ does not admit a Taylor expansion near 0 . This is directly due to the fact that the Cross rheological equation 1 itself is not analytical, since the second term of its development near 0 is of order $n+1$.

[34] It is clear that this exponential law cannot be valid for too thin films. Writing $\eta=E \tau_{r e p}$ (where $E$ is an elastic modulus, and $\tau_{r e p}$ is the reptation time of polymers, the relaxation time of the material), one obtains $\tau_{i}=\tau_{r e p} h / h_{0}$ with $h_{0} \equiv|S| / E$, which leads to a characteristic time $\tau$ smaller than $\tau_{r e p}$ if $h_{i}<h_{0}$ : the relaxation would be too fast.

[35] G. Reiter (private communication)

[36] On the wetting and slippage of polymer films on solid surfaces, see: F. Brochard-Wyart et al., Langmuir 10, 1566 (1994); C. Redon, J. B. Brozoska, and F. BrochardWyart, Macromol. 27, 468 (1994)

[37] An experimental study of the dewetting of PS from poly(methylmethacrylate) on silicon substrates as a function of film thickness was recently proposed. See: C. Wang, G. Krausch, and M. Geoghegan, Langmuir 17, 6269 (2001)

[38] P. Lambooy et al., Phys. Rev. Lett. 76, 1110 (1996); F. Brochard-Wyart, P. Martin, and C. Redon, Langmuir 9, 3682 (1993)

[39] G. Reiter, Eur. Phys. J. E. 8, 251 (2002)

[40] E. Guyon, J.-P. Hulin, and L. Petit, Hydrodynamique Physique, (CNRS Eds., 2nd Ed., 2001), p. 233 